\title{
Mass and Mean Velocity Dispersion Relations for Supermassive Black Holes in Galactic Bulges
}

\author{
Yu-Qing Lou \\ Physics Department and Tsinghua Center for Astrophysics, Tsinghua University, Beijing \\ 100084, China \\ E-mail: louyq@tsinghua.edu.cn \\ Yan-Fei Jiang * \\ Physics Department and Tsinghua Center for Astrophysics, Tsinghua University, Beijing \\ 100084, China \\ E-mail: jiangyanfei1986@gmail.com
}

Growing evidence indicate supermassive black holes (SMBHs) in the mass range of $M_{\mathrm{BH}} \sim 10^{6}-$ $10^{10} M_{\odot}$ lurking in central bulges of many galaxies [1, 2]. Extensive observations reveal fairly tight power laws of $M_{\mathrm{BH}}$ versus the mean stellar velocity dispersion $\sigma$ of the host bulge [3, 4, 5]. The dynamic evolution of a bulge and the formation of a central SMBH should be physically linked by various observational clues. In this contribution, we reproduce the empirical $M_{\mathrm{BH}}-\sigma$ power laws based on a self-similar general polytropic quasi-static bulge evolution [6, 7] and a sensible criterion of forming a SMBH surrounding the central density singularity of a general singular polytropic sphere (SPS) [8]. Other properties of host bulges and central SMBHs are also examined. Based on our $\uparrow$ model, we discuss the intrinsic scatter of the $M_{\mathrm{BH}}-\sigma$ relation and a scenario for the evolution of SMBHs in different host bulges.

self-similar dynamic evolution

VII Microquasar Workshop: Microquasars and Beyond September 1-5 2008

Foca, Izmir, Turkey

*Now at Department of Astrophysical Sciences, Princeton University, Princeton, NJ 08544 USA. 


\section{Supermassive Black Holes and Galactic Host Bulges}

or equivalently

SMBHs form at the centres of elliptical and spiral galaxies [10, 1, 2]. Observationally, SMBH masses $M_{\mathrm{BH}}$ correlate with various properties of spiral galaxy bulges or elliptical galaxies, including bulge luminosities [1, 12, 11], bulge masses $M_{\text {bulge }}$ [12, 11, 13], galaxy light concentrations [14], the Sérsic index of surface brightness profile [15], inner core radii [16], bulge gravitational binding energies [17] and mean stellar velocity dispersions $\sigma$ [18, 19, 5, 3, 4]. These correlations strongly suggest a dynamical link between SMBHs and their host bulges [21, 20].

Among these relations, $M_{\mathrm{BH}}$ and $\sigma$ correlate tightly in power laws with an intrinsic scatter $\lesssim 0.3$ dex [35]. This relation was studied [24, 23, 22] before systematic observations [18, 19] and the emphasis was on outflow effects of galaxies. The idea was further elaborated [25]. A model of singular isothermal sphere (SIS) with rotation [26] was proposed for the $M_{\mathrm{BH}}-\sigma$ relation. This relation was also explored in a semi-analytic model [27] with starbursts while SMBHs being formed and fueled during major mergers. Accretions of collisional dark matter onto SMBHs may also give the $M_{\mathrm{BH}}-\sigma$ relation [28] (see also ref. [29] for a review). There are also numerical simulations to model feedbacks from SMBHs and stars on host galaxies.

There are two empirical types of bulges, namely classical bulges (spiral galaxies with classical bulges or elliptical galaxies) and pseudobulges [30]. While SMBHs in classical bulges are formed after major mergers, pseudobulges do not show apparent merger signatures. Interestingly, pseudobulges also manifest a $M_{\mathrm{BH}}-\sigma$ power law yet with a different exponent [4].

The self-similar quasi-static solutions take the singular polytropic spheres (the static polytropic solutions) as the leading terms. This kind of dynamic solutions has been applied to study the compact stars in a single fluid model [6], galaxy clusters in a two-fluid model [7] and the so-called "champagne flows" in H II regions [9]. In the following, we shall take the quasi-static solutions in a single fluid model to describe host bulges with central SMBHs after long time evolution [8].

\section{A Self-Similar Dynamic Model for $M_{\mathrm{BH}}-\sigma$ Power Laws}

For the dynamic evolution of a galactic bulge, we adopt a few assumptions. First, we treat the stellar bulge as a spherical polytropic fluid as the typical age $\sim 10^{9} \mathrm{yr}$ of galactic bulges is long [31, 32] that they are continuously adjusted. Stellar velocity dispersions produce an effective pressure $P$ against the self-gravity as in the Jeans equation [33]. Secondly, the total mass of the interstellar medium in a galaxy [32] is $\sim 10^{7}-10^{8} M_{\odot}$ only $10^{-2} \sim 10^{-3}$ of the total bulge mass. Although gas densities in broad and narrow line regions of AGNs are high, the filling factor [34] is small $\left(\sim 10^{-3}\right)$ and the gas there may be regarded as condensed clouds. Thus gas is merged into our stellar fluid. Thirdly, the diameter of broad line regions of AGNs [34] is only $\sim 0.1 \mathrm{pc}$ and the disk around a SMBH is even smaller while a galactic bulge size is $\gtrsim 1 \mathrm{kpc}$. We thus ignore small-scale structures around the central SMBH of a spherical bulge. Finally, as the rotation curves of galaxies show [33], the effect of dark matter halo in the innermost region (around several kpcs ) of a galaxy can be neglected. So we do not include the dark matter in awr model when we discuss \begin{tabular}{l|l} 
the dynamic characters of the bulges. & Under the spherical symmetry, \\
\hline
\end{tabular}

$\checkmark h$ Aydrodynamic equations of our model are conservations of mass, radial momentum [6] and 'specific entropy' along streamlines [40, 38, 7]. As bulk flow of stellar fluid is slow, we invoke the 
nonlinear

novel self-similar quasi-static solutions [6, 7] to model the bulge evolution. We use a self-similar transformation [6, 38, 7] to solve general polytropic fluid equations, namely

$r=K^{1 / 2} t^{n} x, \quad \rho=\alpha(x) /\left(4 \pi G t^{2}\right), \quad u=K^{1 / 2} t^{n-1} v(x)$,
dimensionless
parameters $P=K t^{2 n-4} \beta(x) /(4 \pi G), \quad M=K^{3 / 2} t^{3 n-2} m(x) /[(3 n-2) G]$,

where $r$ is the radius and $t$ is time; $x$ is the independent dimensionless similarity variable while $K$ and $n$ are two scaling indices; $G=6.67 \times 10^{-8} \mathrm{~g}^{-1} \mathrm{~cm}^{3} \mathrm{~s}^{-2}$ is the gravity constant; $\rho(r, t)$ is the mass density and $\alpha(x)$ is the reduced mass density; $u(r, t)$ is the radial flow speed and $v(x)$ is the reduced flow speed; $P(r, t)$ is the effective pressure and $\beta(x)$ is the reduced pressure; $M(r, t)$ is the enclosed mass and $m(x)$ is the reduced enclosed mass; reduced variables $\alpha, \beta, v$ and $m$ are $\checkmark$ functions of $x$ only. We require $n>2 / 3$ for a positive mass [6, 7, 38].

By self-similar transformation (1), we readily construct self-similar quasi-static solutions from general polytropic fluid equations, taking the static singular polytropic sphere (SPS) as the leading term. Properties of such asymptotic solutions to the leading order [6, 7] are summarized below. Both initial and eventual mass density profiles scale as $\sim r^{-2 / n}$; accordingly, the bulge enclosed mass profile is $M \propto r^{(3 n-2) / n}$; for either $x \rightarrow 0^{+}$or $x \rightarrow+\infty$, the reduced velocity $v \rightarrow 0$, which means at a time $t$, for either $r \rightarrow 0^{+}$or $r \rightarrow+\infty$ the flow speed $u \rightarrow 0$, or at a radius $r$, when $t$ is short or long enough, the radial flow speed $u \rightarrow 0$. Our model [8] describes a self-similar bulge evolution towards a nearly static configuration after a long time lapse, appropriate for galactic bulges at present epoch. the

As the effective pressure $P$ results from stellar velocity dispersions in the bulge, we readily derive the mean velocity dispersion $\sigma$ in a bulge. By specific entropy conservation along streamlines, we relate $P$ with $\rho$ and $M$ and derive the $P$ profile from our quasi-static solutions [40, 7]. We take the local stellar velocity dispersion as $\sigma_{L}(r, t)=(\gamma P / \rho)^{1 / 2}$ where $\gamma$ is the polytropic index of our stellar fluid. To compare with observations, we derive the spatially averaged stellar velocity dispersion $\sigma$ in the bulge. The bulge boundary is taken as the radius $r_{\mathrm{c}}$ where $\rho$ drops to a value $\rho_{c}$ indistinguishable from the environment. For a class of bulges with same $n, \rho_{c}$ is regarded as a constant for a class of environments. One can show that within $r_{c}$

$$
\sigma=\left[3 n^{1+q / 2} \gamma^{1 / 2} /(4 n-1)\right]\left(4 \pi G \rho_{c}\right)^{(1-n) / 2} A^{3 n q / 4} K^{1 / 2} \equiv \mathscr{Q} K^{1 / 2},
$$

where $q \equiv 2(n+\gamma-2) /(3 n-2)$ and $A \equiv\left\{n^{2-q} /[2(2-n)(3 n-2)]\right\}^{-1 /(n-3 n q / 2)}$.

A SMBH forms at the centre of a galactic bulge that evolves in a self-similar quasi-static manner. Such a SMBH was formed by the collapse of collections of stars and gas towards the bulge centre and grows rapidly by matter accretions at an earlier phase [10]. As the growth timescale for SMBHs is only $\sim 10^{5} \mathrm{yr}$, our quasi-static solutions describe the relatively quiescent phase of galactic bulges after the formation of central SMBHs as a longer history of a bulge evolution. The stellar fluid made up of stars and condensed gas clouds has a slow bulk flow speed towards the central SMBH, sustaining a reservoir of mass accretion for the circumnuclear torus and/or disk. We now introduce the criterion of forming a SMBH. A SMBH mass $M_{\mathrm{BH}}$ and its Schwarzschild radius $r_{\mathrm{s}}$ are related by $M_{\mathrm{BH}}=r_{\mathrm{s}} c^{2} /(2 G)$ where $c$ is the speed of light. As we have pointed out, the bulge enclosed mass is $M \propto r^{(3 n-2) / n}$. If at a radius $r_{\mathrm{s}}, M$ becomes $M=r_{\mathrm{s}} c^{2} /(2 G)$, then a SMBH forms. Only those quasi-static bulges with $n<1$ can form central SMBHs (see Figure 1); 


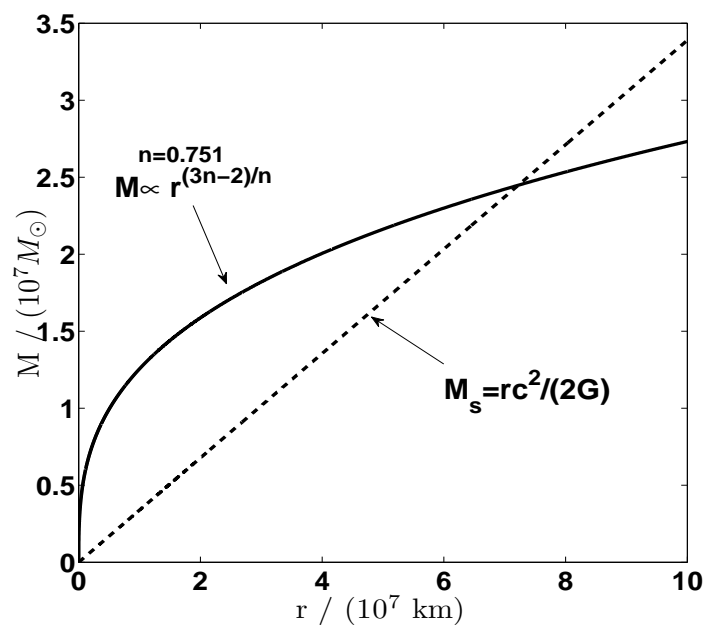

Figure 1: The criterion of forming a central SMBH in a self-similar quasi-static bulge evolution. The enclosed mass power-law is $M \propto r^{0.337}$ with $n=0.751$ (solid curve). Meanwhile, we draw a straight dashed line $M_{\mathrm{s}}=r c^{2} /(2 G)$ for the mass of SMBH versus the Schwarzschild radius $r$. Here at $r_{\mathrm{s}}=7.2 \times 10^{7} \mathrm{~km}$, the straight line intersects the enclosed mass curve for a $2.45 \times 10^{7} M_{\odot} \mathrm{SMBH}$. A self-similar general polytropic quasi-static solution of $n<1 \mathrm{can}$ form a SMBH at the bulge centre by invoking this criterion.

we further derive $M_{\mathrm{BH}} \propto r_{\mathrm{s}} \propto K^{1 /(2-2 n)}$ and the power law below

$$
M_{\mathrm{BH}}=\left(\frac{n A}{3 n-2}\right)^{n /(2-2 n)}\left(\frac{2}{c^{2}}\right)^{3 n-2 /(2-2 n)} \frac{\mathscr{Q}^{1 /(n-1)}}{G} \sigma^{1 /(1-n)} \equiv \mathscr{L} \sigma^{1 /(1-n)},
$$

where $\mathscr{L}$ depends on $c, G, n, \gamma, \rho_{c}$, and the exponent $1 /(1-n)>3$ as $2 / 3<n<1$ is required.

While the $M_{\mathrm{BH}}-\sigma$ power law is very tight with intrinsic scatter $\leq 0.3$ dex for SMBHs and host bulges [35], such scatter is large enough for different fitting parameters [18, 19, 5]. By equation (3), we have a natural interpretation for intrinsic scatter in the observed $M_{\mathrm{BH}}-\sigma$ power law. In our model, all bulges with the same $n$ lie on a straight line with the exponent $1 /(1-n)$ as Figure 2 shows. For a fixed $n$, different bulges are represented by different $K$ values in transformation (1), leading to different $M_{\mathrm{BH}}$ and $\sigma$. However, for bulges with different $n$ values, they lie on different lines. For elliptical galaxies or bulges in spiral galaxies, they appear to eventually take the selfsimilar evolution described above with a certain $n$ value. But pseudobulges may take on different $n$ values. Observationally, we cannot determine a priori the specific $n$ value for a bulge but simply attempt to fit all bulges with a single exponent, which then contributes in part to intrinsic scatter in the observed $M_{\mathrm{BH}}-\sigma$ power law.

To show this, we fit three published $M_{\mathrm{BH}}-\sigma$ power laws in Figure 2. The first one is $M_{\mathrm{BH}}=1.2 \times 10^{8} M_{\odot}\left(\sigma / 200 \mathrm{~km} \mathrm{~s}^{-1}\right)^{3.75}$ given in ref. [19] with our parameters $\left\{n, \gamma, \rho_{c}\right\}$ being $\left\{0.733,1.327,0.47 M_{\odot} \mathrm{pc}^{-3}\right\}$; and the five points correspond to $K=\{0.8,1,2,3,4\} \times 10^{23}$ cgs unit. The second one is $\log \left(M_{\mathrm{BH}} / M_{\odot}\right)=8.13+4.02 \log \left(\sigma / 200 \mathrm{~km} \mathrm{~s}^{-1}\right)$ given in ref. [5] with our parameters $\left\{n, \gamma, \rho_{c}\right\}$ being $\left\{0.7512,1.33,0.0122 M_{\odot} \mathrm{pc}^{-3}\right\}$; and the seven points correspond to $K=\{1,2,4,6,8,10,20\} \times 10^{22}$ cgs unit. The third one is $\log \left(M_{\mathrm{BH}} / M_{\odot}\right)=8.28+$ 
host bulge

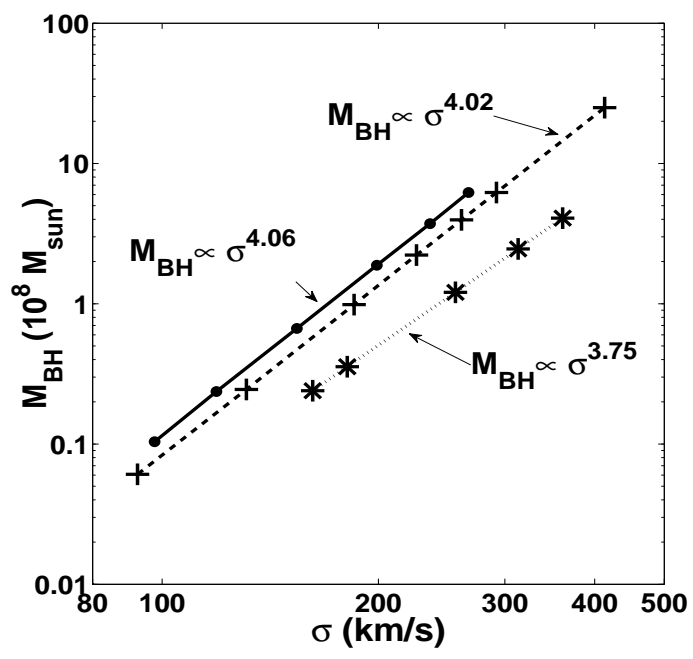

Figure 2: Power-law $M_{\mathrm{BH}}-\sigma$ relations according to our general polytropic model for quasi-static selfsimilanevolution. Three index values $n=0.7537,0.7512,0.7333$ are adopted for three different kinds (solid [4], dashed [5], dotted lines [19]) of $M_{\mathrm{BH}}-\sigma$ power laws given by equation (3). Given $n$, we calculate the SMBH mass $M_{\mathrm{BH}}$ and the mean stellar velocity dispersion $\sigma$ for a certain $K$ value in transformation (1). A bulge has different $\sigma$ for different $K$. Each bulge-SMBH system is represented by a point in this display. All systems of same $n$ give a straight line, while systems of different $n$ correspond to different straight lines here.

$4.06 \log \left(\sigma / 200 \mathrm{~km} \mathrm{~s}^{-1}\right)$ given in ref. [4] with our parameters $\left\{n, \gamma, \rho_{c}\right\}$ being $\{0.7537,1.332$, $\left.0.00364 M_{\odot} \mathrm{pc}^{-3}\right\}$; and the six points correspond to $K=\{0.6,0.9,1.5,2.5,3.5,4.5\} \times 10^{23} \mathrm{cgs}$ unit. Clearly, to fit all these points in Figure 2 with a single power law, we would get a different result with higher intrinsic scatter. In the three fitting examples, bulge inflow speeds of stellar fluid are slow $\left(\sim 0.1-1 \mathrm{~km} \mathrm{~s}^{-1}\right)$, a feature of our self-similar quasi-static solutions. Near the SMBH boundary $r_{\mathrm{s}}$, the inflow rest mass-energy flux falls in the range of $10^{40}-10^{45} \mathrm{erg} \mathrm{s}^{-1}$ in these examples, sufficient to supply the observed X-ray luminosities [36]. There can be outgoing accretion shocks around a SMBH in these flows. As the age of galactic bulges [31] is so long $\left(\sim 10^{9} \mathrm{yr}\right)$ that such shocks should have gone outside bulges.

Besides the $M_{\mathrm{BH}}-\sigma$ relation, observations reveal $M_{\mathrm{BH}} \propto M_{\text {bulge }}^{1.12}$ with $M_{\text {bulge }}$ being the bulge mass [13] and $M_{\mathrm{BH}} \propto E_{\mathrm{g}}^{0.6}$ with $E_{\mathrm{g}}$ being the absolute value of the bulge gravitational binding energy [17]. Using our criterion of forming a SMBH and the bulge radius $r_{c}$, we derive a power law between $M_{\mathrm{BH}}$ and $M_{\text {bulge }}$ as $M_{\mathrm{BH}} \propto M_{\text {bulge }}^{1 /(3-3 n)}$. For $n=0.75$, our result leads to relations in ref. [26] but for a nonisothermal general SPS. The bulge gravitational binding energy, without contributions from dark matter halo and a disk [17], is $E_{\mathrm{g}} \approx \int_{0}^{r_{c}} G M \rho 4 \pi r d r$. For self-similar quasi-static solutions, we obtain $M_{\mathrm{BH}} \propto E_{\mathrm{g}}^{1 /(5-5 n)}$.

As another class of bulges, pseudobulges are believed to have formed without merging in contrast to classical bulges. Nonetheless, pseudobulges follow a $M_{\mathrm{BH}}-\sigma$ power law but with a different exponent [37, 4]. In ref. [4], this $M_{\mathrm{BH}}-\sigma$ relation is found to be $\log \left(M_{\mathrm{BH}} / M_{\odot}\right)=7.5+$ $4.5 \log \left(\sigma / 200 \mathrm{~km} \mathrm{~s}^{-1}\right)$. This conclusion is natural according to our model in that pseudobulges 
may take a different self-similar quasi-static evolution for a different $n$ value. Due to their different formation history, they show a different $M_{\mathrm{BH}}-\sigma$ power law as observed. For $\left\{n, \gamma, \rho_{c}\right\}$ being $\left\{0.7778,1.34,0.000426 M_{\odot} \mathrm{pc}^{-3}\right\}$, our model can also fit this power law.

\section{Conclusions and Discussion}

The tight $M_{\mathrm{BH}}-\sigma$ power laws and other relations among the SMBH mass $M_{\mathrm{BH}}$ and known properties of host bulges strongly suggest coeval growths of SMBHs and galactic bulges [39, 29, [27]. In our model, while forming a Schwarzschild SMBH at the bulge centre (e.g., by collapse of gas and stars or by merging), the spherical general polytropic bulge evolves in a self-similar quasi-static phase for a long time. We then reproduce empirical $M_{\mathrm{BH}}-\sigma$ power laws. Different energetic processes appear to give rise to different scaling index $n$ values.

Besides classical bulges and pseudobulges, there are also 'core' elliptical galaxies, thought to have formed by 'dry' mergers. A steeper $M_{\mathrm{BH}}-\sigma$ relation exists in these galaxies as compared to that for classical bulges [16]. This can be accommodated in our scenario that all hosts of SMBHs may finally evolve into self-similar quasi-static phase with different scaling parameters (e.g., different $n$ for the slope and different $\rho_{c}$ for the normalization of the $M_{\mathrm{BH}}-\sigma$ relation). We thus provide a unified framework to model the the relatively quiescent evolution phase of SMBH host bulges and SMBH masses. As the observed $M_{\mathrm{BH}}-\sigma$ relation for classical bulges is tight, the elliptical galaxies and spiral galaxies appear to take close $n$ values for merging processes.

In our model, $n$ is a key scaling index to determine the exponent of the $M_{\mathrm{BH}}-\sigma$ power law. The smaller the value of $n$ is, the steeper the profile of the density is and the smaller the index of the $M_{\mathrm{BH}}-\sigma$ relation is. If we think the SMBHs are formed by collapse of stars and gas and a less steeper density distribution may provide a more effective mechanism to form SMBHs, then we conclude that for a certain value of velocity dispersions, the smaller the mass of the initially formed $\mathrm{SMBH}$ is, the smaller the value of $n$ is.

that

\section{Acknowledgement}

This research was supported in part by Tsinghua Center for Astrophysics (THCA), National Science Foundation of China (NSFC) grants 10373009 and 10533020, the Yangtze Endowment and SRFDP 20050003088 of Ministry of Education, and NBSTTP J0630317 of NSFC at Tsinghua University. The kind hospitality of Institut für Theoretishce Physik und Astrophysik der ChristianAlbrechts-Universität Kiel" is gratefully acknowledged.

\section{References}

[1] Kormendy, J. \& Richstone, D. Inward bound - the search for supermassive black holes in galactic nuclei. Annu. Rev. Astron. Astrophys. 33, 581-624 (1995)

[2] Kormendy, J. Coevolution of black holes and galaxies. Obs. Astrophys. Ser. 1, 1 (2004)

[3] Ferrarese, L. \& Ford, H. Supermassive blackholes in galactic nuclei: past, present and future research. Space Sci. Rev. 116, 523-624 (2005) 
[4] $\mathrm{Hu}, \mathrm{J}$. The black hole mass-stellar velocity dispersion correlation: bulges versus pseudo-bulges. Mon. Not. Roy. Astron. Soc. in press (2008), (arxiv: 0801.1481v3) 386, 2242-2252

[5] Tremaine, S. et al. The slope of the black hole mass versus velocity dispersion correlation. Astrophys. J. 574, 740-753 (2002)

[6] Lou, Y.-Q. \& Wang, W. G. New self-similar solutions of polytropic gas dynamics. Mon. Not. Roy. Astron. Soc. 372, 885-900 (2006)

[7] Lou, Y.-Q., Jiang, Y. F. \& Jin, C. C. Self-similar shocks and winds in galaxy clusters. Mon. Not. Roy. Astron. Soc. 386, 835-858 (2008)

[8] Lou, Y.-Q. \& Jiang, Y. F. Supermassive black holes in galactic bulges. Mon. Not. Roy. Astron. Soc. Letter, in pross (2008) (2008arXiv:0809.1126L) 391, L44-L48

[9] Hu, R. Y. \& Lou, Y.-Q. Self-similar polytropic champagne flows in H II regions. Mon. Not. Roy. Astron. Soc., in puess (2008) (2008arXiv0808.2090H) 390, 1619-1634

[10] Lynden-Bell, D. Galactic nuclei as collapsed old quasars. Nature 223, 690-695 (1969)

[11] Marconi, A. \& Hunt, L. K. The relation between black hole mass, bulge mass, and near-infrared luminosity $M_{\mathrm{BH}}-\sigma$ and mass relations. Astrophys. J. 589, L21-24 (2003)

[12] Magorrian, J. et al. The demography of massive dark objects in galaxy centres. Astron. J. 115, 2285-2312 (1998)

[13] Häring, N. \& Rix, H.-W. On the black hole massícbulge mass relation. Astrophys. J. 604, L89-92 (2004)

[14] Graham, A. W., Erwin P., Caon N. \& Trujillo I., A correlation between galaxy light concentration and supermassive black hole mass. Astrophys. J. 563, L11-14 (2001)

[15] Graham, A. W. \& Driver, S. P. A log-quadratic relation for predicting supermassive black hole masses from the host bulge sersic index. Astrophys. J. 655, 77 (2007)

[16] Lauer, T. R. et al. Masses of nuclear black holes in luminous elliptical galaxies and implications for the space density of the massive black holes. Astrophys. J. 662, 808-834 (2007)

[17] Aller, M. C. \& Richstone, D. O. Host galaxy bulge predictors of supermassive black hole mass. Astrophys. J. 665, 120-156 (2007)

[18] Ferrarese, L. \& Merritt, D. A fundamental relation between supermassive black holes and their host galaxies. Astrophys. J. 539, L9-12 (2000)

[19] Gebhardt, K. et al. A relationship between nuclear black hole mass and galaxy velocity dispersion. Astrophys. J. 539, L13-16 (2000)

[20] Li, Y., Haiman, Z. \& Low, M. M. Correlations between central massive objects and their host galaxies: from bulgeless spirals to ellipticals. Astrophys. J. 663, 61-70 (2007)

[21] Springel, V., Matteo, T. D. \& Hernquist, L. Modelling feedback fromstars and black holes ingalaxy mergers. Mon. Not. Roy. Astron. Soc. 361, 776-794 (2005)

[22] Blandford, R. D. Origin and evolution of massive black holes in galactic nuclei. Galaxy Dynamics, ASP Conf. Ser. 182, 87-95 (1999)

[23] Fabian, A. C. The obscured growth of massive black holes. Mon. Not. Roy. Astron. Soc. 308, L39-43 (1999)

[24] Silk, J. \& Rees, M. J. Quasars and galaxy formation. Astron. Astrophys. 331, L1-L4 (1998) 
[25] King, A. Black holes, galaxy formation, and the $M_{\mathrm{bh}}-\sigma$ relation. Astrophys. J. 596, L27-29 (2003)

[26] Adams, F. C., Graff, D. S. \& Richstone, D. O. A theoretical model for the $M_{\mathrm{bh}}-\sigma$ relation for supermassive black holes in galaxies. Astrophys. J. 551, L31-35 (2001)

[27] Kauffmann, G. \& Haehnelt, M. A unified model for the evolution of the galaxies and quasars. Mon. Not. Roy. Astron. Soc. 311, 576-588 (2000)

[28] Ostriker, J. P. Collisional dark matter and the origin of massive black holes. Phys. Rev. Lett. 84, 5258-5260 (2000)

[29] Haehnelt, M. G. Coevolution of Black Holes and Galaxies, from the Carnegie Observatories Centennial Symposia, 405 (Cambridge Univ. Press, 2004)

[30] Drory, N. \& Fisher, D. B. A connection between bulge properties and the bimodality of galaxies. Astrophys. J. 664, 640-649 (2007)

[31] Frogel, J. A. The galactic nuclear bulge and the stellar content of spheroidal systems. Ann. Rev. Astron. Astrophys. 26, 51-92 (1988)

[32] Gnedin, N. Y., Norman, M. L. \& Ostriker, J. P. Formation of galactic bulges. AIPC 470, 48-57

[33] Binney, J. \& Tremaine, S. Galactic Dynamics (Princeton University Press, 1994)

[34] Osterbrock, D. E. \& Ferland, G. J. Astrophysics of Gaseous Nebulae and Active Galactic Nuclei (University Science Books, 2rd Edition, 2006)

[35] Novak, G. S., Faber, S. M. \& Dekel, A. On the correlations of massive black holes with their host galaxies. Astrophys. J. 637, 96-103 (2006)

[36] Komossa, S. et al. Discovery of superstrong, fading, iron line emission and double-peaked balmer lines of the galaxy SDSS J095209.56214313.3: The light echo of a huge flare. Astrophys. J. 678, L13-16 (2008)

[37] Kormendy, J. \& Gebhardt, K. The 20th Texas Symposium on Relativistic Astrophysics, 363 (2001) in H. Martel, J.C. Wheeler, eds.

[38] Lou, Y.-Q. \& Cao, Y. Self-similar dynamics of a relativistically hot gas. Mon. Not. Roy. Astron. Soc. 384, $611(2008)$

[39] Page, M. J., Stevens, J. A., Mittaz, J. P. D. \& Carrera, F. J. Submillimeter evidence for the coeval growth of massive black holes and galaxy bulges. Science 294, 2516-2518 (2001)

[40] Wang, W. G. \& Lou, Y.-Q. Self-similar dynamics of a magnetized polytropic gas. Astropys. Space Sci. 311, 363 (2007)

[41] Wang, W. G. \& Lou, Y.-Q. Dynamic evolution of a quasi-spherical general polytropic magnetofluid with self-gravity. Astropys. Space Sci. 315, 135 (2008) 